\title{
Advance of Molecular Imaging Technology and Targeted Imaging Agent in Imaging and Therapy
}

\author{
Zhi-Yi Chen, ${ }^{1}$ Yi-Xiang Wang, ${ }^{2}$ Yan Lin, ${ }^{1}$ Jin-Shan Zhang, ${ }^{3}$ Feng Yang, \\ Qiu-Lan Zhou, ${ }^{1}$ and Yang-Ying Liao ${ }^{1}$ \\ ${ }^{1}$ Department of Ultrasound Medicine, Laboratory of Ultrasound Molecular Imaging, The Third Affiliated Hospital of Guangzhou \\ Medical University, Guangzhou 510150, China \\ ${ }^{2}$ Department of Imaging and Interventional Radiology, Prince of Wales Hospital, The Chinese University of Hong Kong, \\ Shatin, New Territories, Hong Kong \\ ${ }^{3}$ Department of Nuclear Medicine, The Third Affiliated Hospital of Guangzhou Medical University, Guangzhou 510150, China
}

Correspondence should be addressed to Zhi-Yi Chen; winchen@vip.126.com

Received 27 October 2013; Revised 29 December 2013; Accepted 30 December 2013; Published 13 February 2014

Academic Editor: Weibo Cai

Copyright (C) 2014 Zhi-Yi Chen et al. This is an open access article distributed under the Creative Commons Attribution License, which permits unrestricted use, distribution, and reproduction in any medium, provided the original work is properly cited.

\begin{abstract}
Molecular imaging is an emerging field that integrates advanced imaging technology with cellular and molecular biology. It can realize noninvasive and real time visualization, measurement of physiological or pathological process in the living organism at the cellular and molecular level, providing an effective method of information acquiring for diagnosis, therapy, and drug development and evaluating treatment of efficacy. Molecular imaging requires high resolution and high sensitive instruments and specific imaging agents that link the imaging signal with molecular event. Recently, the application of new emerging chemical technology and nanotechnology has stimulated the development of imaging agents. Nanoparticles modified with small molecule, peptide, antibody, and aptamer have been extensively applied for preclinical studies. Therapeutic drug or gene is incorporated into nanoparticles to construct multifunctional imaging agents which allow for theranostic applications. In this review, we will discuss the characteristics of molecular imaging, the novel imaging agent including targeted imaging agent and multifunctional imaging agent, as well as cite some examples of their application in molecular imaging and therapy.
\end{abstract}

\section{Introduction}

Molecular imaging is a rapidly developed multidiscipline which involves molecular biology, chemistry, computer, engineering, and medicine [1]. It can realize noninvasive and real time visualization, measurement of physiological or pathological process in the living organism at the cellular or molecular level $[2,3]$. And it also allows repeated studies in the same animal, thus making it possible to collect longitudinal data and reduce the number of animals and cost [4]. Therefore, molecular imaging plays an important role in earlier detection, accurate diagnosis, and drug development and discovery [5-7]. Molecular imaging requires high resolution and high sensitive instruments to detect specific imaging agents that link the imaging signal with molecular event [8]. There are five imaging modalities available for molecular imaging, including X-ray computed tomography imaging (CT), optical imaging (OI), radionuclide imaging (involving PET and SPECT), ultrasound (US) imaging and magnetic resonance imaging (MRI) [9]. In the past two decades, imaging instruments have grown exponentially. Improvement in instruments and iterative image reconstruction has resulted in high resolution images that reveal tiny lesion and realize accurate quantification of biological process. A parallel development has been the preparation of imaging agents which can bind their targets with high specificity and affinity [10]. In this review, we will discuss the characteristics of molecular imaging, some novel imaging agents based on nanoparticles including targeted imaging agent and multifunctional imaging agents, and cite some examples of their application in molecular imaging and therapy.

\section{Molecular Imaging Technology}

2.1. Radionuclide Imaging. Radionuclide molecular imaging including PET and SPECT is the earliest and most mature 
molecular imaging technique. Due to its advantages of high sensitivity and quantifiability, radionuclide molecular imaging plays an important role in clinical and preclinical researches [11]. Over the past decade, with the progress of molecular biology and radiochemistry, a variety of tracer with high specificity and affinity appeared. A lot of preclinical and clinical studies have confirmed the feasibility of using radionuclide molecular imaging to detect tumor and predict response to therapy $[12,13]$.

2.1.1. PET. PET is the molecular imaging modality most extensively used in current clinic routine. It measures the signal originated from the radioactive decay of neutron-deficient radioisotopes (such as ${ }^{11} \mathrm{C},{ }^{15} \mathrm{O},{ }^{18} \mathrm{~F}$, and ${ }^{131} \mathrm{I}$ ) that are intravenously injected into the body. These isotopes emit positrons which are ejected from the nucleus as a result of springless interactions with electrons in surrounding tissue. The positrons rapidly lose kinetic energy by spreading around the tissue and collide with an electron to form two $511 \mathrm{keV}$ photons which are taking trajectory $180^{\circ}$ apart, and this is an event known as annihilation [14]. A PET detector surrounding the subject is designed to detect the signal and convert the resulting electrical signal into sinograms that are finally rebuilt into tomographic images.

Because of its high sensitivity of $10^{-11} \sim 10^{-12} \mathrm{~mol} / \mathrm{L}$, limitless depth of penetration, and quantitative capabilities, PET becomes a powerful tool for clinical diagnosis and basic research including neurology, cardiology, and particularly oncology $[15,16]$. In the clinic, PET is crucial for cancer detection and staging, as well as evaluation of response to therapy. A mass of radiotracer has been employed for cancer imaging, with ${ }^{18} \mathrm{~F}$-FDG being the key one. The main disadvantage of PET is the lack of anatomical parameters to identify molecular events with accurate correlation to anatomical findings, and this disadvantage has recently been compensated by merging these devices with either CT or MRI [17]. It is reported that whole-body PET/CT improves the accuracy of cancer diagnosis and staging. With the widespread of instrument, PET/CT has become an important tool for predicting therapeutic response, providing useful information for the decision to stop ineffective treatment or switch to a more efficient treatment. It is shown that up to $40 \%$ of patients with cancer have changed the treatment because of application of PET/CT [18]. Recently, Andrade et al. [19] evaluated 40 patients with invasive ductal breast carcinomas that received neoadjuvant chemotherapy by FDG-PET/CT. They found that the application of FDG-PET/CT after the second course of NAC could predict therapeutic response in ductal breast carcinomas and potentially recognise the nonresponding patients for whom ineffective chemotherapy should be avoided. Other limitations of PET are their safety profile and the requirement of cyclotron to produce radiopharmaceuticals.

2.1.2. SPECT. Unlike PET, SPECT directly detects gammaray photon emitted by the chosen radionuclide during their decay. Compared with PET, SPECT is more affordable and extensively employed in the clinical routine, but it is generally

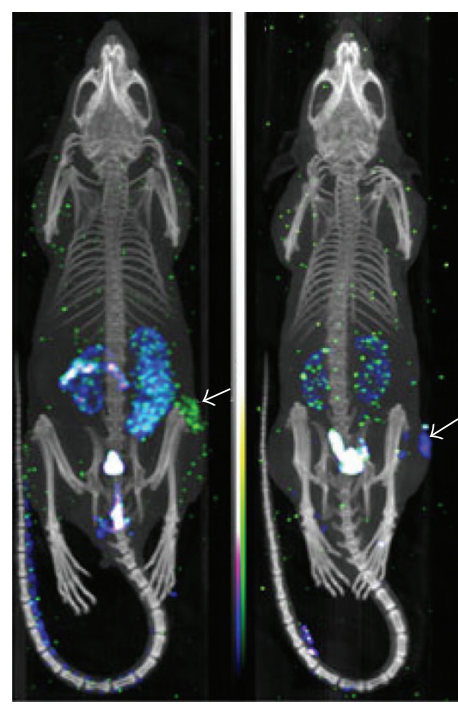

(a)

(b)

FIGURE 1: Dual-isotope SPECT/CT images of tumor-bearing mice. (a) The mice were imaged $1 \mathrm{~h}$ after coinjection of $1.32 \mathrm{nmol}$ ${ }^{177} \mathrm{Lu}$-DOTA_cRGDfK (yellow-green colormap) and $1.32 \mathrm{nmol}$ ${ }^{111}$ In-DOTA - cRADfK (blue-purple colormap); (b) the mice were imaged $1 \mathrm{~h}$ after coinjection of $1.32 \mathrm{nmol}^{111} \mathrm{In}$-DOTA-cRGDfK (blue-purple colormap) and $1.32 \mathrm{nmol}{ }^{177} \mathrm{Lu}-\mathrm{DOTA}-\mathrm{cRADfK}$ (yellow-green colormap). The white arrows indicate the locations of tumor [23].

less sensitive since the photons which are not traveling along the axis of the collimator are rejected by the scanner. The spatial resolution $(8-10 \mathrm{~mm})$ of SPECT is lower than that of clinical PET (5-7 mm) [20], but the spatial resolution of small animal SPECT (micro-SPECT) is higher than that of PET due to the development of imaging equipment. Thus micro-SPECT is more available in preclinical investigations including the transformation research and animal studies such as oncology, neurology, cardiovascular disease, and drug development [21, 22]. Additionally, since the radionuclides commonly available for SPECT have longer half-life periods (ranging from a few hours to days), longitudinal studies can be performed. Based on the isotope-specific energies of the emitted photons (e.g., $\left[{ }^{111} \mathrm{In}\right]$ indium: 171 and $245 \mathrm{keV} ;\left[{ }^{177} \mathrm{Lu}\right]$ lutetium: 202 and $307 \mathrm{keV}$ ), SPECT can distinguish different radioisotopes, therefore making it possible to image different targets simultaneously. Hijnen et al. [23] took advantage of this characteristic and devised a dual-isotope experiment on a micro-SPECT system. In their study, they quantified the biodistribution and tumor uptake of the angiogenesis tracer cRGD via SPECT (Figure 1). However, dual-tracer images can be severely distorted due to cross talk between the two isotopes. Recently, Hapdey et al. [24] put forward a generalized spectral factor analysis (GSFA) method for solving this problem in simultaneous ${ }^{99 \mathrm{~m}} \mathrm{Tc} /{ }^{123} \mathrm{I}$ SPECT, which proved that simultaneous ${ }^{99 \mathrm{~m}} \mathrm{Tc} /{ }^{123} \mathrm{I}$ imaging can provide images of similar quantitative accuracy through GSFA as when using sequential and scatter-free ${ }^{99 \mathrm{~m}} \mathrm{Tc} /{ }^{123} \mathrm{I}$ imaging in brain SPECT. 
2.2. Magnetic Resonance Imaging. Magnetic resonance imaging (MRI) is a highly versatile imaging modality [25]. During the past decades, improvement in instrument launched the field of MRI into a new era of molecular imaging. The merits of MRI as an imaging modality for molecular imaging are relatively high temporal and spatial resolution, excellent tissue contrast and tissue penetration, no ionizing radiation, noninvasiveness for serial studies, and simultaneous acquisition of anatomical structure and physiological function [26]. Nevertheless, molecular MRI is limited by its relatively low sensitivity, and this requires the introduction of imaging agent and development of powerful signal amplification strategies. Imaging agent design is hence an important topic in molecular MRI. Currently, the MR imaging agents are mainly divided into two kinds: ferromagnetism contrast agents and paramagnetic contrast agents. The former is considered as negative contrast agents which mainly reduce the signal in T2-weighted images, while the latter is referred to as positive contrast agents that increase the signal in T1-weighted images. The most representative negative contrast agents are superparamagnetic iron oxide (SPIO) and ultrasmall superparamagnetic iron oxide (USPIO), and typical positive contrast agents are small molecular weight compounds involving a single Lanthanide chelate as signal producing element (e.g., gadolinium-DTPA). To date, there are numerous examples of MR molecular imaging which have confirmed the potential of this technology. Rapley et al. [27] demonstrated that the application of antibody-SPIO complex and MRI was a feasible method for detecting and measuring nucleosome concentration in vitro, which was expected to become a diagnostic, prognostic, and predictive tool in the management of cancer. Recently, Debergh et al. [28] showed that the application of a small monogadolinated tracer targeting $\alpha_{\mathrm{v}} \beta_{3}$ integrin and MR molecular imaging was a promising strategy for evaluation of colorectal cancers associated angiogenesis.

2.3. X-Ray Computed Tomography Imaging. CT imaging technologies have undergone a very fast development in the last years. High resolution small animal CT (microCT) has transformed CT imaging from organ, tissue to molecular level, which is playing an increasingly important role in preclinical researches $[29,30]$. The main advantages of CT are high spatial resolution (micro-CT is $0.02 \sim 0.30 \mathrm{~mm}$; clinical CT is $0.5 \sim 2.0 \mathrm{~mm}$ ), fast acquisition time, relative simplicity, availability, excellent hard-tissue imaging. Due to limitations like ionizing radiation, limited soft tissue resolution, and poor sensitivity $\left(10^{-2} \sim 10^{-3} \mathrm{~mol} / \mathrm{L}\right)$, CT is always combined with other imaging modalities such as SPECT, PET to provide anatomical parameters for the biochemical and physiological findings [31]. Recently, the development of CT contrast agents has brought new hope for CT molecular imaging [32]. For example, Hyafil et al. [33] reported cellular imaging of macrophage in the atherosclerotic plaque in a rabbit model through CT with iodinated nanoparticles (N1177) (Figure 2). Pan et al. [34] found that polymeric nanoparticles contrast agents which were comprised of organometallics or radiopaque organically soluble elements could further improve the imaging sensitivity for CT. Also, targeted gold nanoparticles for CT imaging at the cellular and molecular level have been successfully prepared. Li et al. [35] conjugated 2-deoxy-D-glucose (2-DG) to a gold nanoparticle to prepare CT molecular imaging agent (AuNP-2-DG) which could be used for CT imaging to obtain high resolution anatomic structure and metabolic information of tumor. The results of in vitro experiments proved that AuNP-2-DG could be used as a functional CT contrast agent. However, though the findings from these CT molecular imaging experiments show attractive prospect, further study is required to explore the feasibility of CT molecular imaging.

2.4. Optical Imaging. Optical molecular imaging technology is an emerging technology, based on genomics, proteomics, and modern optical technology. At present, the most widely used optical molecular imaging modalities in vivo include bioluminescence imaging (BLI) and fluorescence imaging. As optical imaging is performed through the use of light, thus it is considered as relatively safe. And due to their advantages of high sensitivity and low cost, optical imaging plays a central role in the investigation of tumor occurrence, progressions and relevant drug development $[36,37]$. The main disadvantages of optical imaging are that the depth of penetration is limited as the energy of photons is low, thereby making it nearly impossible to image deep tissues in large subjects. Due to the small size of experimental animals, the depth of penetration is not a problem for them. Therefore, optical imaging is widely used for preclinical research.

BLI is taking advantage of the light produced by the enzymatic oxidation reaction of luciferase and its substrate. The luciferase is usually generated from the reporter gene. As opposed to fluorescence imaging, the light source of BLI is from interior. And since tissue do not produce endogenous bioluminescence, the sensitivity and signal to background is better than fluorescence imaging. BLI is widely used to observe the biological processes in vivo like disease progression, certain gene expression, tumor growth, and metastasis and evaluate the effect of certain treatments. For example, Niu et al. [38] introduced a caspase-3 specific cyclic firefly luciferase reporter gene (pcFluc-DEVD) into UM-SCC-22B and $4 \mathrm{~T} 1$ cells then induced apoptosis of the cells by different concentrations of doxorubicin and evaluated the effect by BLI. The results exhibited that BLI intensity was increased as early as $24 \mathrm{~h}$ after treatment and achieved a maximum at around day 12 , which indicated that BLI with pcFluc-DEVD as a reporter gene can help to observe the kinetics of the apoptotic process in a real-time manner (Figure 3).

Fluorescence imaging is a versatile technique with a number of strengths such as relative low cost and multiplexed imaging. As light is absorbed by hemoglobin and other molecules, the depth of penetration is limited (usually $<1 \mathrm{~cm}$ ). Hence, fluorescence imaging is mainly used in animal researches. Atreya et al. [39] took advantage of the feature that integrin $\alpha_{\mathrm{v}} \beta_{3}$ and vascular endothelial growth factor receptor (VEGFR) are overexpressed in tumor; they used the integrin $\alpha_{\mathrm{v}} \beta_{3}$ fluorescent probe to detect the tumor angiogenesis in nude mice. The result showed there were strong fluorescence signals for $\alpha_{\mathrm{v}} \beta_{3}$ in vivo via a fluorescent scanner, which 


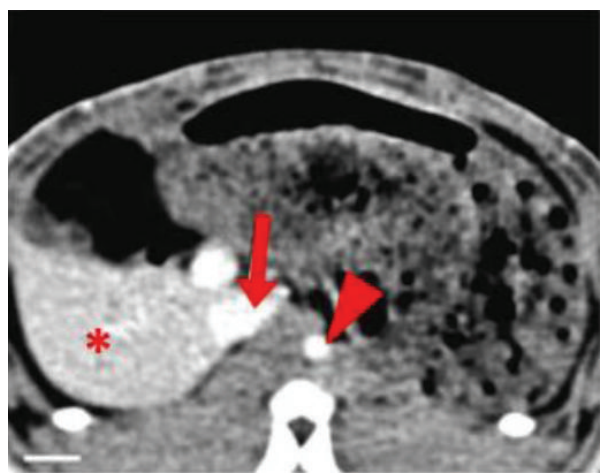

(a)

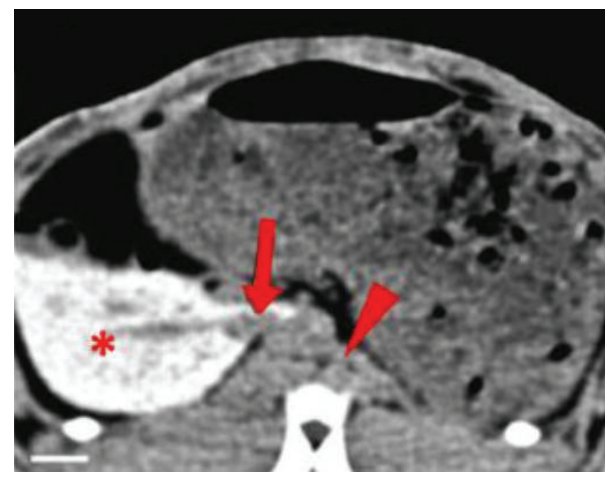

(c)

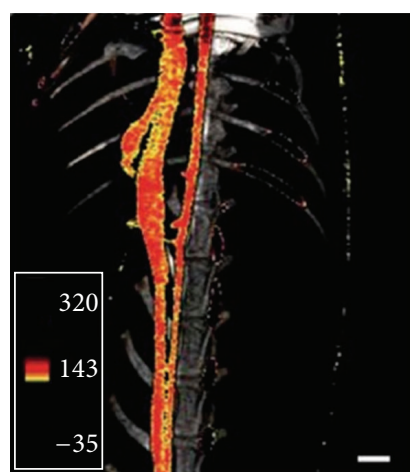

(b)

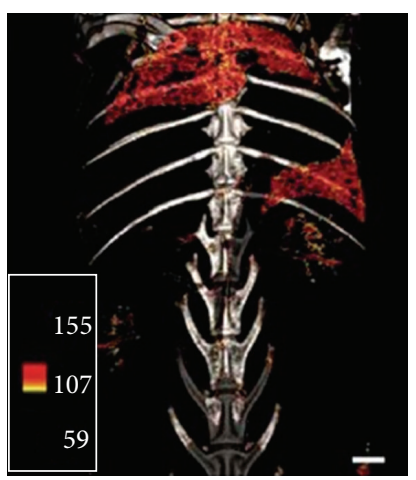

(d)

FIGURE 2: CT molecular imaging based on iodinated nanoparticles (N1177). The kinetics and distribution of iodinated nanoparticles (N1177) in the atherosclerotic rabbit model were displayed by CT imaging. (a) 5 min after intravenous injection of N1177, CT image display aorta, and vena cava (red arrow) with a noticeable signal; (b) the reconstruction of three-dimensional CT imaging; (c) $2 \mathrm{~h}$ after intravenous injection of N1177, a strong signal was displayed in the spleen $(*)$; (d) use of color scale to reconstruct three-dimensional CT angiograms of CT scan. (adopted from Hyafil et al. [33]).

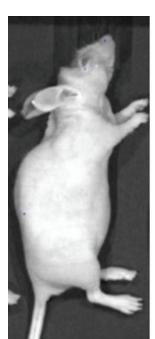

$0 \mathrm{hr}$

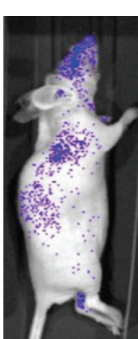

$24 \mathrm{hr}$

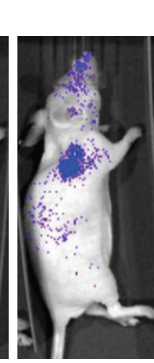

$48 \mathrm{hr}$

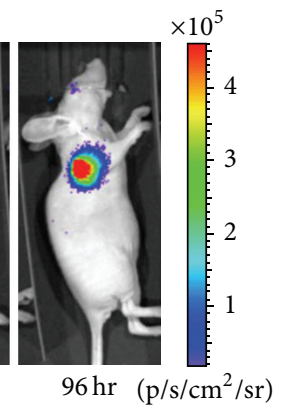

Figure 3: After doxorubicin treatment ( $5 \mathrm{mg} / \mathrm{kg}$ ), bioluminescence imaging was performed to observe caspase activation in $22 \mathrm{~B}$ pcFluc-DEVD (adopted from Niu et al. [38]).

indicated angiogenesis increased in the cancer tissue. Also, they proved that the targeted visualization of VEGF could be achieved via a fluorescent-labeled antibody against the VEGFR (Figure 4).

2.5. Ultrasound Molecular Imaging. With the use of ultrasound contrast agent, ultrasound imaging enables specific and sensitive depiction of molecular targets [40,41]. Compared with other molecular imaging modalities, ultrasound molecular imaging has many advantages including good temporal resolution, quantitative data, real-time practice, noninvasiveness, relatively inexpensive cost, and no ionizing radiation. In addition, it is a unique modality in some sense that it can be employed for diagnostic imaging and as a therapeutic tool $[42,43]$. Mancini et al. [44] proved that the use of ultrasound molecular imaging with microbubble targeting VEGFR-2 might be a valuable method for noninvasively detecting and quantifying of VEGFR-2 expression in thyroid cancer in mice, and it is also a useful tool for differentiating benign from malignant thyroid nodules.

Besides, for diagnostic purposes, molecular ultrasound imaging can also be applied for theranostic purposes. This is mainly achieved by loading gene or drug onto microbubble. The interaction between ultrasound and microbubble results in stable and inertial cavitation, which can transiently increase vascular and cellular membrane permeability, thereby potentially augments the extravasation and/or take up of drugs or genes loaded on microbubble. Li et al. [45] prepared a novel microbubble carrying $10-\mathrm{HCPT}$, and they demonstrated that the injection of 10-HCPT loaded microbubble and exposure to ultrasound could increase the drug concentration in tumor remarkably, leading to a significant increase in tumor inhibition rate (70.6\%) compared with $10-\mathrm{HCPT}$ loaded microbubble alone (47.8\%) as well as commercial 


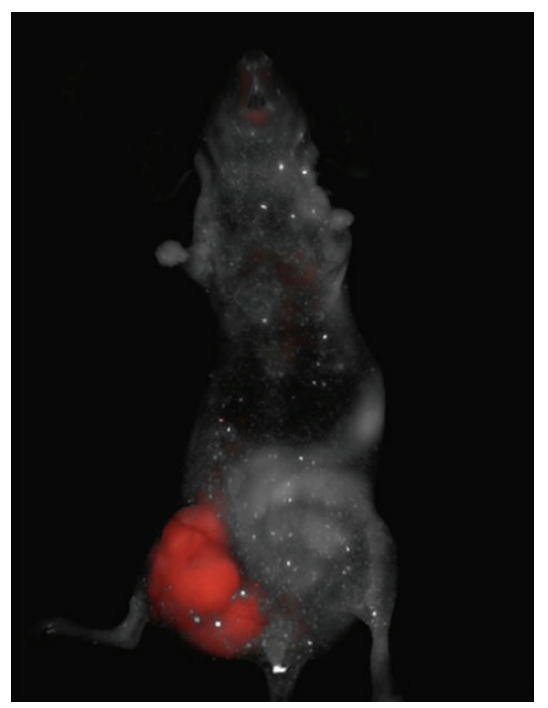

(a)

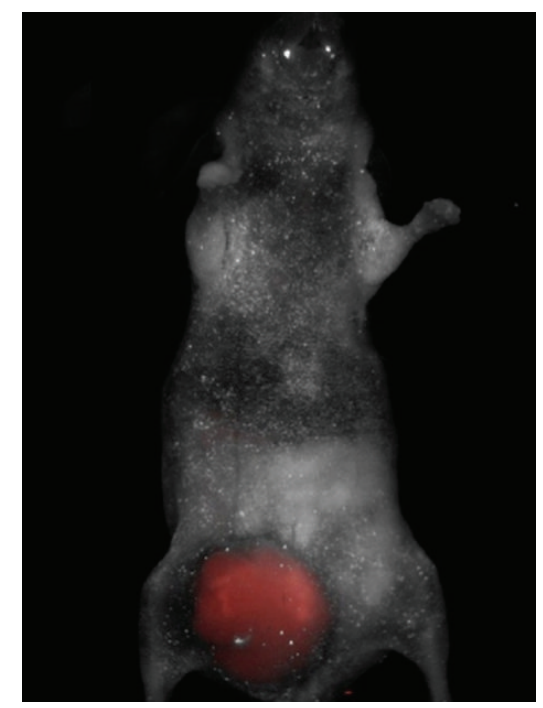

(b)

FIGURE 4: Nude mice was injected with SW620 CRC cells then administered intravenously with integrin $\alpha_{\mathrm{v}} \beta_{3}$ fluorescent probe after the formation of intestinal tumor. Via a fluorescent scanner, the targeted fluorescence signals for $\alpha_{\mathrm{v}} \beta_{3}$ were observed in vivo (a). A fluorescentlabeled antibody against the VEGFR was injected intravenously, and fluorescence was detected using a multispectral in vivo imaging system (b) (adopted from Atreya et al. [39]).

HCPT injection (49.4\%). Our studies have confirmed that the combination of ultrasound and microbubble could significantly increase the transfection efficacy [46].

2.6. Multimodality Imaging. Among all molecular imaging techniques, every molecular imaging technique has its advantages and disadvantages. No single one is perfect and enough to provide comprehensive information for disease diagnosis [47]. In general, CT, MRI, and US are anatomic imaging methods but they have low sensitivity. Radionuclide imaging and optical imaging are functional imaging techniques, while they suffer from low resolution, which often lack structural parameter. The combination of different molecular imaging techniques, namely, multimodality imaging, can provide synergistic advantages over any modality alone and compensate for the disadvantages of each imaging system while taking advantage of their individual strengths, which has become the developmental trend of modern medical image now [48-51]. Multimodality imaging such as PET/SPECT, PET/CT, and PET/MRI can be used to obtain anatomical and molecular information while providing enough information for clinical diagnosis [52]. For example, the combination of PET and CT can produce coregistered data providing regions of increased ${ }^{18}$ F-FDG accumulation on the PET image with precise correlation to anatomical findings' locations on the CT scan, thus the specificity and sensitivity of PET in detecting lesion are enhanced, so as the accuracy in delineating target volume. Abgral et al. [53] conducted a study that included 91 patients who once had HN cancer but were cured and without any clinical evidence of recurrence to assess the diagnostic capabilities of ${ }^{18} \mathrm{~F}$-FDG PET/CT in these patients. The results showed that the sensitivity, specificity, and accuracy of ${ }^{18} \mathrm{~F}$ FDG PET/CT for the diagnosis of $\mathrm{HN}$ cancer recurrence were
$100 \%, 85 \%$, and $90 \%$, respectively. The positive predictive value was $77 \%$, while the negative predictive value was $100 \%$.

MRI shows obvious advantages over CT including excellent soft tissue contrast, high spatial resolution, and no ionizing radiation; thus studies of PET/MRI become the focus of concern and have achieved initial progress. Lee et al. [54] used PET/MRI to evaluate the presence of monocytes in nonischemic remote zone after myocardial infarction, which proved that inflammatory cells of infarct zone and distant noninfarcted area could be observed by PET/MRI images. Recently, PET/MRI has been applied to clinic. Kjær et al. [55] performed a PET/MRI examination on a female patient with cervical cancer for restaging following radiotherapy and compared the results with PET/CT, which showed that $\mathrm{PET} / \mathrm{MRI}$ performed well compared to PET/CT. It had a more precise definition of the primary tumor (Figure 5). However, PET with MRI in a single gantry which is dedicated to simultaneous PET/MRI is technically more challenging.

In addition to the combination of PET and CT or MRI, other multimodality imaging methods have been reported, such as OI/US, OI/CT, OI/MRI, and US/MRI [56-58]. OI is a versatile technique in preclinical research as it possesses the advantages of high specificity and sensitivity, low cost, relatively high-flux, and convenience. Ultrasound, on the other hand, has strength of high resolution. The strengths of these imaging techniques are complementary. The combination of them, which takes advantage of high sensitivity of optical imaging and high resolution of ultrasound, may produce synergistic effect and provide images with good spatial and temporal resolution. Lately, Li et al. [56] designed a system which integrated 3D ultrasound imaging and 3D continuous transillumination fluorescence tomography. They evaluated the feasibility and performance of the system by using phantoms 


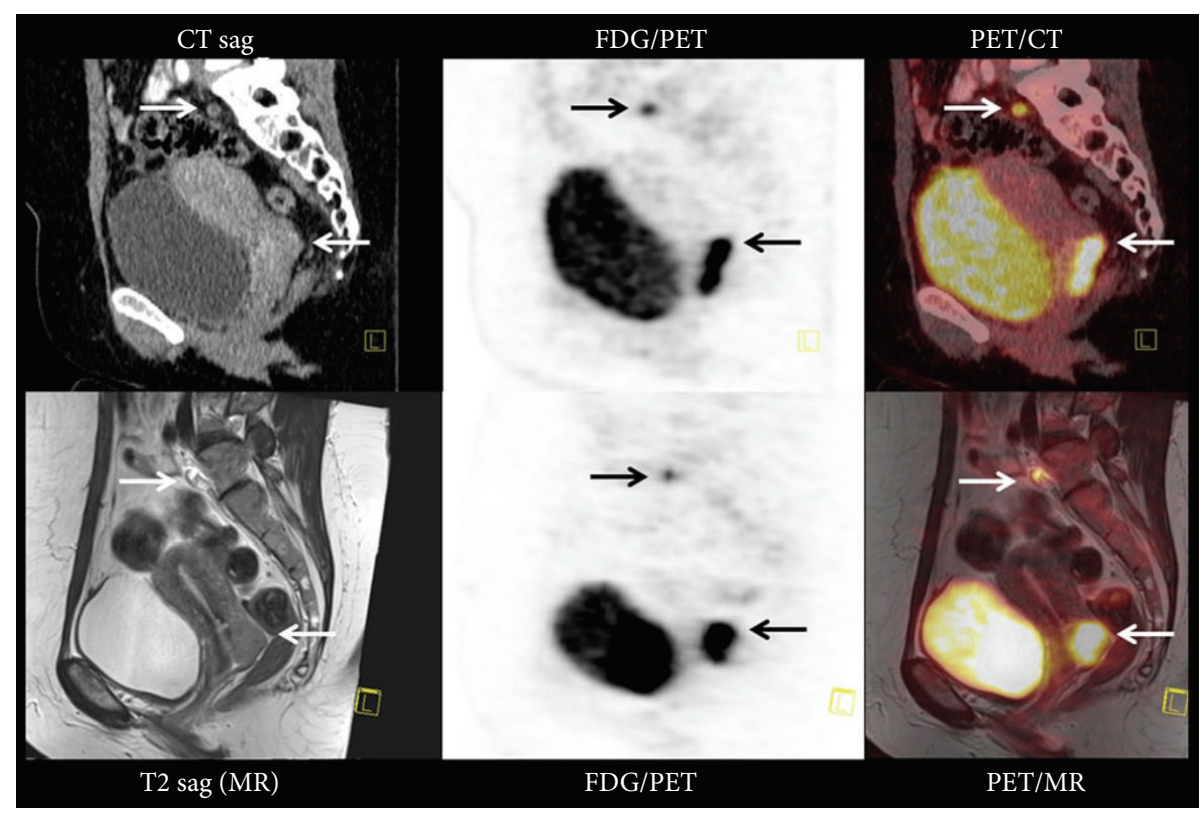

FIGURE 5: 31-year-old female patient with cervical cancer had an ${ }^{18} \mathrm{~F}$ FDG-PET/CT and PET/MRI examination for restaging following radiotherapy. PET/CT showed the primary tumour in the cervix behind the urine bladder and a lymph node in the pelvis, both indicated with arrows. PET/MR exhibited the same findings but with a more precise definition of the primary tumor (adopted from Kjær et al. [55]).

and atherosclerosis disease mouse model. Results proved that ultrasound structural images could improve the quality of the fluorescent image reconstruction and the activity of VCAM could be detected through the system, which showed a great potential for the application in in vivo molecular imaging. As this system could provide structural information while keeping the merits of low cost of fluorescence imaging, it is worthy of promotion and application for the future.

Therefore, it can be seen that multimodality imaging is not a simple addition of various imaging technologies but rather for producing synergistic effect. It can provide more information to understand the biological processes comprehensively and objectively. But it is still difficult to carry out multimodality imaging due to existing problems regarding the accuracy of coregistered image, extra ionizing radiation, the extra dosage of contrast agent, and the toxicity of fused contrast agents. Therefore, it is in urgent need of developing multimodality molecular imaging agent.

\section{Imaging Agents}

Molecular imaging depends greatly on the development of specific and sensitive imaging agents, which is a pivotal ratelimiting step in the development of molecular imaging [8]. In a molecular imaging study, imaging agents are mainly used for interrogating or coupling back about a specific target of interest [10]. They usually consist of signal component and targeting component. In recent years, the advancement of biochemistry has been achieved and the development of molecular imaging technologies has led to the production of a mass of molecular imaging agents [57]. A mass of targeting moieties, such as small molecule, peptide, antibody, and aptamer, is applied to decorate ligand-directed ("targeted") imaging agents to recognise specific pathological tissues [9]. Furthermore, nanoparticles with unique properties have emerged as a promising class of molecule imaging agents. Targeting moieties, therapeutic drug or gene, and different imaging labels can be incorporated into nanoparticles to construct targeted imaging agents and multifunctional imaging agents which allow for multimodal imaging and theranostic applications [58, 59].

\subsection{Targeted Molecular Imaging Agents Based on Small} Molecules. Small molecules, of which the size is usually less than $500 \mathrm{Da}$, are playing an important role in molecular imaging. Due to their small size, small molecules have a wide range of application including intracellular and central nervous system. ${ }^{18} \mathrm{~F}$-FDG is the most widely used imaging agent based on small molecules, which is clinically applied for cancer imaging [60]. Ren et al. [61] reported the use of gadolinium (Gd) to label uniformly phosphorothioate-modified human tumor telomerase reverse transcriptase (hTERT) antisense oligonucleotide (ASON) targeting hTERT mRNA and conducted a study in BALB/c nude mice with 7.0T MicroMRI, showing that Gd-DOTA-ASON was a potential intracellular MR contrast probe for detection of telomerasepositive carcinomas (Figure 6).

3.2. Targeted Molecular Imaging Agents Based on Peptide. Peptide is an important class of ligand used for molecular imaging [62]. Compared to small molecules, peptide has many advantages, such as superior selectivity and specificity and easier modification without changing their binding properties or distribution. Additionally, peptide is more stable in atmospheric temperature than antibodies and has a lower immunogenicity compared with antibodies. Recently, 


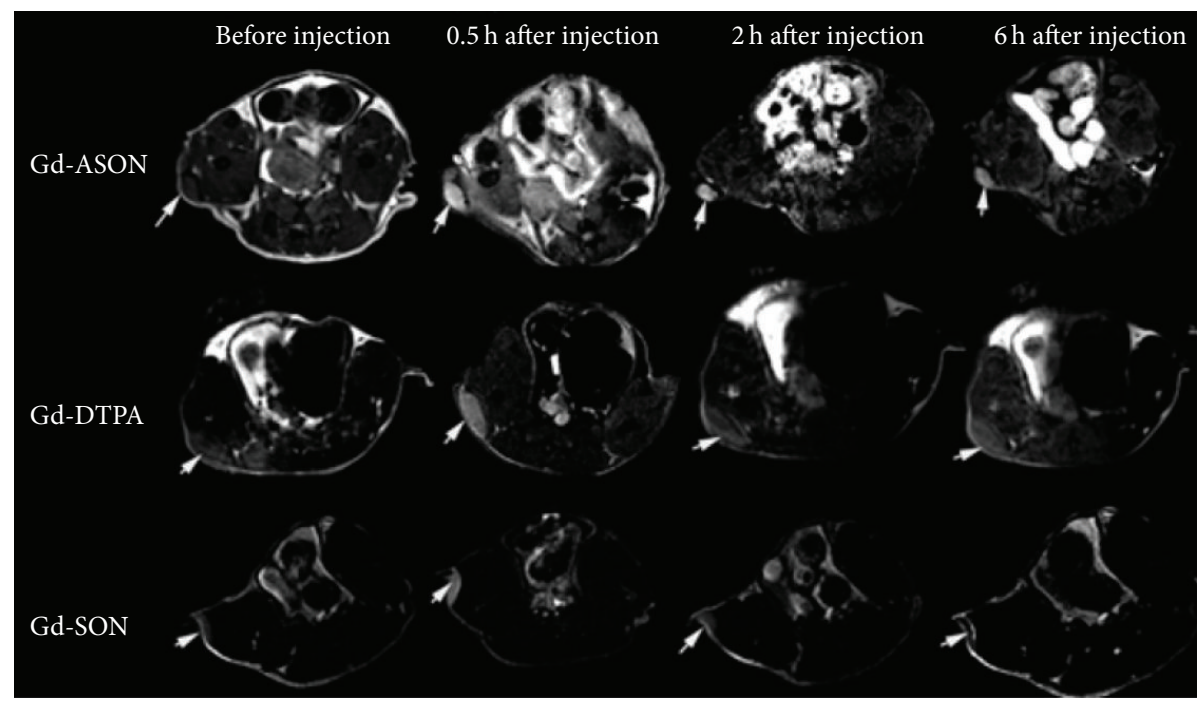

FIGURE 6: T1-weighted multiple slice multiple echo weighted MRI of nude mice bearing A549 tumors before and 0.5, 2, and 6h after intraperitoneal injection. Tumors (arrows) all showed different enhancement level at $0.5 \mathrm{~h}$, but in Gd-DOTA-ASON (upper line), the enhancement remained at $6 \mathrm{~h}$, while Gd-DTPA (middle line) decreased obviously from $2 \mathrm{~h}$ (repetition time, $561 \mathrm{~ms}$, echo time, $14 \mathrm{~ms}$, and field of view, $4 \mathrm{~cm}$ ); Gd-DOTA-SON (lower line) showed a similar trend to that of Gd-DOTA-ASON but with lower enhancement (adopted from Ren et al. [61]).

Hansen et al. [63] conjugated a peptide which could bind to the urokinase plasminogen activator receptor (uPAR) with high specificity and affinity to polyethylene glycol (PEG) coated USPIO nanoparticles. In vitro result showed that the peptide conjugated USPIO nanoparticles had a five times higher uptake in a uPAR positive cell line compared to the nanoparticles with a nonspecific peptide. Hackel et al. [64] used ${ }^{18} \mathrm{~F}$ labelled 2 cystine knot peptides and performed micro-PET on BxPC3 pancreatic adenocarcinoma xenografts in mice with them. Results indicated that these cystine knot peptide tracers had high tumor uptake, showing translational promise for cancer imaging.

3.3. Targeted Molecular Imaging Agents Based on Antibodies. Characterized by the ability to bind their target with high specificity and affinity and easy synthesis, antibodies have been applied for diagnosis and therapy [65]. So far, there are more than eight FDA-approved radiolabeled antibodies approved for SPECT molecular imaging and about twenty antibodies approved for therapy. Recently, Zhang et al. [66] reported the use of PET with (61/64) Cu-NOTA-TRC105Fab to image CD105 expression, which showed obvious and target-specific uptake in the 4T1 tumor (Figure 7). Abdolahi et al. [67] conjugated superparamagnetic iron oxide nanoparticles with an antibody which could bind to the extracellular domain of PSMA and performed MRI with it. Results demonstrated that the nanoprobe could act as a specific MRI contrast agent for detection of PSMA-expressing prostate cancer cells.

3.4. Targeted Molecular Imaging Agents Based on Aptamer. Aptamer, single-stranded DNA or RNA oligonucleotides, can bind to their target with high selectivity and specificity
[68]. Because they enjoy a number of merits including high affinity and specificity, low immunogenicity, small size, stable structures, and ease of production, aptamers have recently attracted increasing attention $[69,70]$. In recent years, probe based on aptamer provides a new strategy for molecular imaging. Shi et al. [71] reported the use of an activatable aptamer probe (AAP) which could bind membrane proteins of living cancer cells with a high degree of specificity to visualize the cancer inside mice. The results showed that the AAP could be used to specifically, quickly detect CCRFCEM cell in the serum or CCRF-CEM cell tumor in mice. Bagalkot et al. [72] recently described the use of a novel quantum dot conjugated with prostate specific membrane antigen (PSMA) RNA aptamers to image and cure cancer. Aptamer conjugated nanoparticles provide new strategy for targeted multimodality imaging. Hwang et al. [73] described a multimodal cancer-targeted imaging system for simultaneous fluorescence imaging, radionuclide imaging, and MRI in vivo through an aptamer conjugated nanoparticle probes. The probe was synthesized by the AS1411 aptamer (MF-AS1411) which targeted nucleolin a cobalt-ferrite nanoparticle comprised core of fluorescent rhodamine and a silica-based shell. Then the probe was injected intravenously into nude mice bearing tumour xenografts and performed ${ }^{67} \mathrm{Ga}$ radionuclide imaging and MRI. The SPECT image showed that this probe exhibited a better accumulation in tumour $24 \mathrm{~h}$ after injection than the control group. Furthermore, MRI images showed the AS1411 conjugated nanoparticle as black signal in tumours $24 \mathrm{~h}$ after injection, while there were no T2 negative signals in the control group. Finally, fluorescence images exhibited a higher signal in tumours of mice injected with aptamer conjugated nanoparticles compared to the control one. Taken together, these results proved that aptamers can deliver the nanoparticles to tumor tissue (Figure 8). 

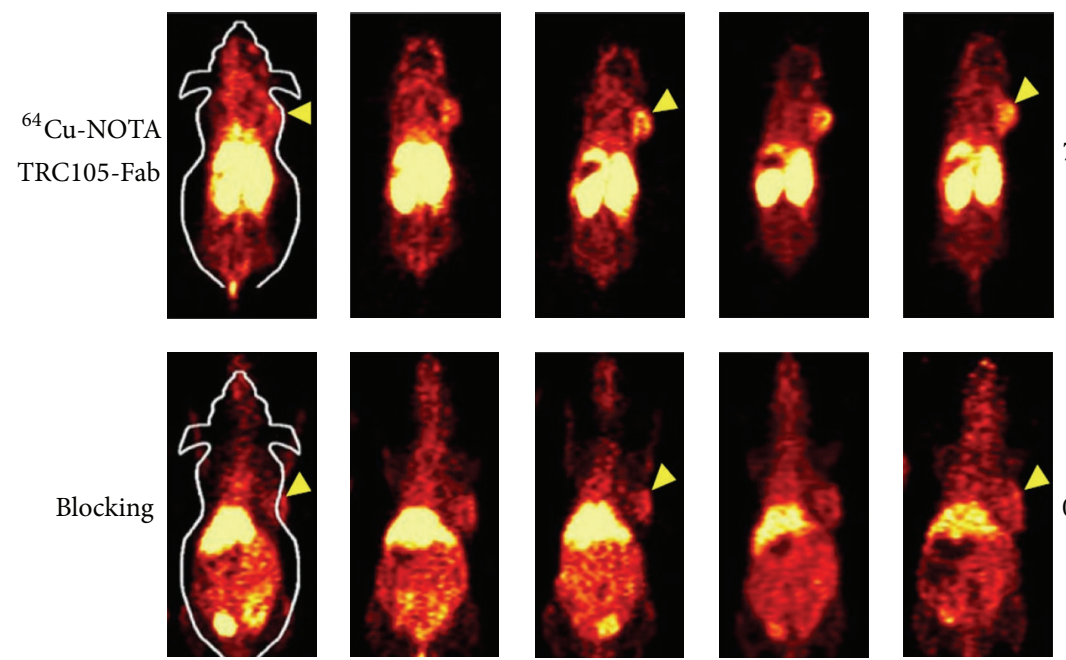

$2 \mathrm{~h}$

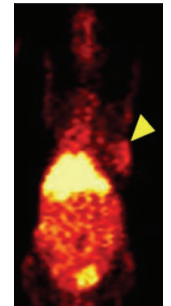

$5 \mathrm{~h}$

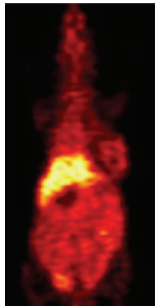

$16 \mathrm{~h}$

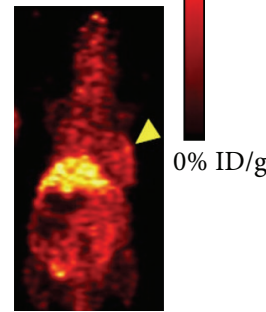

$24 \mathrm{~h}$

FIGURE 7: PET images were performed at $0.5,2,5,16$, and $24 \mathrm{~h}$ p.i. of ${ }^{64} \mathrm{Cu}$-NOTA-TRC105-Fab, or ${ }^{64} \mathrm{Cu}-\mathrm{NOTA}-\mathrm{TRC105}$-Fab after treatment with a $2 \mathrm{mg}$ blocking dose of TRC105 before injection (adopted from Zhang et al. [66]).

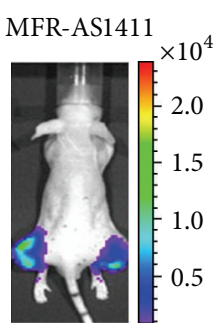

(a1)

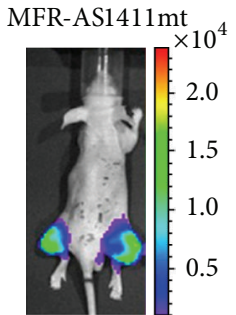

(a2)

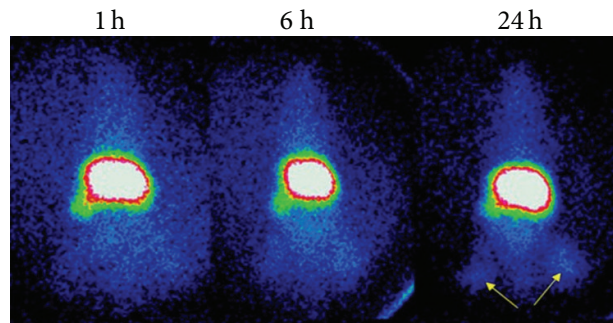

(b1)

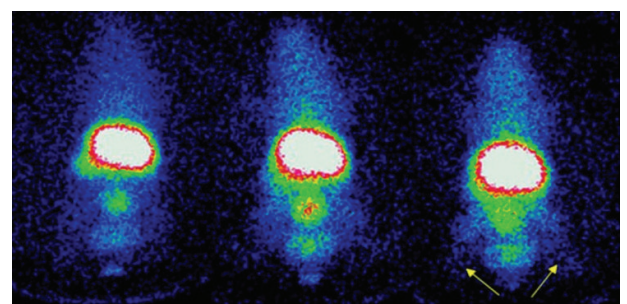

(b2)

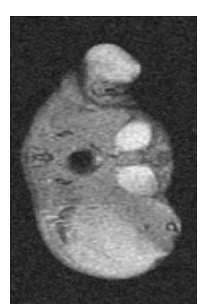

(c1)

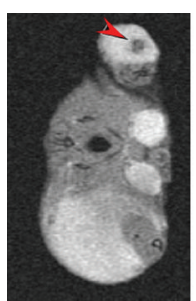

(c2)

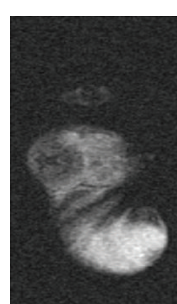

(d1)

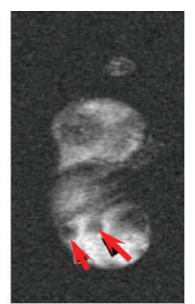

(d2)

FIGURE 8: Cancer-targeted multimodality imaging through MFRAS1411 conjugated nanoparticles. Tumor-bearing mice were intravenously injected with MFR-AS1411 nanoparticles (b1) and MFR-AS1411mt (b2) nanoparticles (control group). Radionuclide images were performed at 1, 6, and $24 \mathrm{~h}$ after injection. Scintigraphic images of tumors in mice injected with MFRAS1411 exhibited that MFR-AS1411 nanoparticles were accumulated in the tumors but MFRAS1411mt were not. Tumor growth patterns were followed using bioluminescence signals acquired from luciferase-expressing C6 cells (a1, a2). MR images of tumor-bearing mice before (c1, d1) and after (c2, d2) injection of MFR-AS1411 were obtained. Dark signal intensities at tumor sites were detected in MFR-AS1411-injected mice (arrowhead) (adopted from Hwang et al. [73]).

3.5. Multifunctional Molecular Imaging Agent. With multimodality imaging techniques clearly on the rise, the development over these new techniques has led to explosive growth in multimodal imaging agent researches $[74,75]$. Since different imaging techniques can only detect their corresponding contrast agents, the patient who intends to perform multimodality imaging may need to inject different contrast mediums. This will increase the patient's economic burden and the additional stress on the blood clearance mechanisms. Moreover, it will expose him to side effect by the mutual interference between different contrast agents. Therefore, many researches are trying to design and develop a probe which can be detected by different imaging modalities so as to boost the clinical benefits of multimodality imaging. Due to their limited attachment points, small molecules are not suitable for multimodality imaging. On contrast, nanoparticles are attractive candidates for multimodal imaging probe [76-78]. They possess high surface areas to volume ratio, which allows multiple modifications to ligands and different imaging agents within the nanoparticles or on its surface. Recently, John et al. [77] described the use of a targeted multimodal protein-shell microsphere which contained iron oxide nanoparticles in their cores and conjugated with the RGD peptide ligand to enhance the imaging ability of ultrasound, 
MRI and MM-OCT. Also, Liu et al. [79] designed an iron oxide nanoparticle-embedded polymeric microbubble used for ultrasound imaging and MRI. Liang et al. [80] reported the synthesis and evaluation of streptavidin nanoparticlebased complexes which were functionalized with biotinylated anti-Her2 Herceptin antibody as multimodality imaging agents to detect tumor in a model via both SPECT system and IVSI fluorescence camera. Willmann et al. [81] prepared a polymeric contrast agent MBQDs - PLGA suited for optical and ultrasonic molecular imaging. In addition, they used PET to assess in vivo whole-body biodistribution of microbubbles functionalized with anti-VEGFR2 antibodies that were marked by the radiofluorination agent N-succinimidyl-4$\left[{ }^{18} \mathrm{~F}\right]$ fluorobenzoate (SFB) [82]. Hence, it can be seen that multimodal imaging agents are available, but their clinical application still need further research.

Apart from imaging agent and ligands, therapeutic drug or gene can also be incorporated in nanoparticles to construct theragnostic agent which have an important role in therapy. This agent enables observation of the extent of disease prior to therapeutic intervention. The ability to identify disease status and controlled delivery of drug using the same agent would help ensure that only potential responsive patients would be treated. Therefore, theragnostic agents show a promising prospective in therapy. Yan et al. [83] have synthesized a novel targeted drug-loaded microbubble functionalized with LyP1 , a breast tumor homing peptide, and evaluated its effect in vivo. Results of the study demonstrated that the LyP1-coated PTX-loaded microbubble improved the antitumor efficacy markedly and had great potential in ultrasoundassisted breast cancer treatment.

\section{Prospect}

Molecular imaging technology truly enables dynamical, quantitative visualization of specific biochemical activity without trauma in vivo at cellular and molecular level. It has a direct impact on the modern and future medicine. In recent years, molecular imaging technology has seen certain progresses in the early diagnosis, curative effect monitoring of diseases, drug development, gene therapy, and other fields, but some key problems regarding theory, technology, and system, especially molecular imaging agents and imaging equipment, are not solved yet. The development of a new probe is not straightforward. Due to barriers in delivery, biological compatibility, and the diversity between species, there are only a few of clinical molecular imaging agents available currently. With the development of technology, it is expected that more advancement will be achieved in the area of molecular imaging agent including targeted molecular imaging agent and multifunctional molecular imaging agents. The application of nanoparticles has provided a platform for theranostic researches.

Additionally, although a number of molecular imaging instruments are available, all have their limitations. For instance, MRI is a useful clinical diagnostic tool, but it suffers from somewhat poor sensitivity. Therefore, more focus should be performed on refining and improving upon existing instrumentation and further efforts geared toward the combination of different modalities so as to make up the disadvantages of different instruments. Multimodality imaging has many advantages, but some problems still exist and need to be solved, such as the difficulty of designing a PET/MRI system suited for the entire body, cost increase, performance improvement, and multimodal contrast agent fusion.

Furthermore, as an emerging interdisciplinary science which brings together nuclear medicine, ultrasonic medicine, radiology, pharmacy, and materials science, the development of molecular imaging needs to strengthen interdisciplinary cooperation. Awareness of importance of multidisciplinary joint research should be strengthened.

The rapid expansion of molecular imaging application shows a promising prospect. Although overall the molecular imaging is still at the initial stage of development, we believe that within the support and cooperation from imaging experts and scholars, molecular imaging techniques would eventually realize clinical transformation.

\section{Conflict of Interests}

The authors declare no conflict of interests regarding the publication of this paper.

\section{Acknowledgments}

This work was supported by National Natural Science Foundation of China (no. 81371572), Research Fund of Doctoral Program of Universities (no. 20124401120002), Sixth Special Project of China Postdoctoral Science Foundation (no. 2013T60826), China Postdoctoral Science Foundation (no. 2011M501375), Research Project of Guangzhou Technology Bureau (no. 12C22021645), Research Project of Science and Technology Guangdong Province (no. 2012B050300026), Research Project of Science and Technology Liwan District (no. 20124414124), and Natural Science Foundation of Guangdong Province (no. S2012040006593).

\section{References}

[1] D. Pan, G. M. Lanza, S. A. Wickline, and S. D. Caruthers, "Nanomedicine: perspective and promises with ligand-directed molecular imaging," European Journal of Radiology, vol. 70, no. 2, pp. 274-285, 2009.

[2] E. A. Osborn and F. A. Jaffer, "Advances in molecular imaging of atherosclerotic vascular disease," Current Opinion in Cardiology, vol. 23, no. 6, pp. 620-628, 2008.

[3] R. Weissleder and M. J. Pittet, "Imaging in the era of molecular oncology," Nature, vol. 452, no. 7187, pp. 580-589, 2008.

[4] R. Atreya and M. Goetz, "Molecular imaging in gastroenterology," Nature Reviews Gastroenterology \& Hepatology, vol. 10, no. 12, pp. 704-7712, 2013.

[5] S. E. P. New and E. Aikawa, "Molecular imaging insights into early inflammatory stages of arterial and aortic valve calcification," Circulation Research, vol. 108, no. 11, pp. 1381-1391, 2011. 
[6] C. M. Gomes, A. J. Abrunhosa, P. Ramos, and E. K. J. Pauwels, "Molecular imaging with SPECT as a tool for drug development," Advanced Drug Delivery Reviews, vol. 63, no. 7, pp. 547$554,2011$.

[7] A. T. Byrne, A. E. O’Connor, M. Hall et al., "Vascular-targeted photodynamic therapy with BF2-chelated tetraaryl-azadipyrromethene agents: a multi-modality molecular imaging approach to therapeutic assessment," British Journal of Cancer, vol. 101, no. 9, pp. 1565-1573, 2009.

[8] S. Luo, E. Zhang, Y. Su, T. Cheng, and C. Shi, "A review of NIR dyes in cancer targeting and imaging," Biomaterials, vol. 32, no. 29, pp. 7127-7138, 2011.

[9] M. L. James and S. S. Gambhir, "A molecular imaging primer: modalities, imaging agents, and applications," Physiological Reviews, vol. 92, no. 2, pp. 897-965, 2012.

[10] K. Chen and X. Chen, "Design and development of molecular imaging probes," Current Topics in Medicinal Chemistry, vol. 10, no. 12, pp. 1227-1236, 2010.

[11] C. J. Anderson and R. Ferdani, "Copper-64 radiopharmaceuticals for PET imaging of cancer: advances in preclinical and clinical research," Cancer Biotherapy and Radiopharmaceuticals, vol. 24, no. 4, pp. 379-393, 2009.

[12] J. Yuan, H. Zhang, H. Kaur et al., "Synthesis and characterization of theranostic poly(HPMA)-c(RGDyK)-DOTA-64Cu copolymer targeting tumor angiogenesis: tumor localization visualized by positron emission tomography," Molecular Imaging, vol. 12, no. 3, pp. 203-212, 2012.

[13] C. Xavier, I. Vaneycken, M. D’huyvetter et al., "Synthesis, preclinical validation, dosimetry, and toxicity of 68Ga-NOTAAnti-HER2 nanobodies for iPET imaging of HER2 receptor expression in cancer," The Journal of Nuclear Medicine, vol. 54, no. 5, pp. 776-784, 2013.

[14] M. E. Phelps, "Positron emission tomography provides molecular imaging of biological processes," Proceedings of the National Academy of Sciences of the United States of America, vol. 97, no. 16, pp. 9226-9233, 2000.

[15] A. Afshar-Oromieh, U. Haberkorn, M. Eder, M. Eisenhut, and C. Zechmann, "PET imaging with a [68Ga]gallium-labelled PSMA ligand for the diagnosis of prostate cancer: biodistribution in humans and first evaluation of tumour lesions," European Journal of Nuclear Medicine and Molecular Imaging, vol. 40, no. 4, pp. 486-495, 2013.

[16] A. J. Beer, H. Kessler, H. J. Wester et al., "PET imaging of integrin $\alpha 3 \beta$ v expression," Theranosttiics, vol. 1, pp. 48-57, 2011.

[17] B. J. Pichler, M. S. Judenhofer, and C. Pfannenberg, "Multimodal imaging approaches: PET/CT and PET/MRI-part 1," Handbook of Experimental Pharmacology, no. 185, pp. 109-132, 2008.

[18] D. Belkić and K. Belkić, "Molecular imaging in the framework of personalized cancer medicine," The Israel Medical Association Journal, vol. 15, pp. 665-672, 2013.

[19] W. P. Andrade, E. N. Lima, C. A. Osório et al., "Can FDG-PET/ CT predict early response to neoadjuvant chemotherapy in breast cancer?" European Journal of Surgical Oncology, vol. 39, no. 12, pp. 1358-1363, 2013.

[20] P. Blake, B. Johnson, and J. W. VanMeter, "Positron emission tomography (PET) and single photon emission computed tomography (SPECT): clinical applications," Journal of NeuroOphthalmology, vol. 23, no. 1, pp. 34-41, 2003.

[21] A. M. Catafau and S. Bullich, "Molecular imaging PET and SPECT approaches for improving productivity of antipsychotic drug discovery and development," Current Medicinal Chemistry, vol. 20, no. 3, pp. 378-388, 2013.

[22] M. Benadiba, G. Luurtsema, and L. Wichert-Ana, "New molecular targets for PET and SPECT imaging in neurodegenerative diseases," Revista Brasileira de Psiquiatria, vol. 34, supplement 2, pp. S125-S148, 2012.

[23] N. M. Hijnen, A. de Vries, K. Nicolay, and H. Grüll, "Dualisotope111In/177Lu SPECT imaging as a tool in molecular imaging tracer design," Contrast Media and Molecular Imaging, vol. 7, no. 2, pp. 214-222, 2012.

[24] S. Hapdey, M. Soret, and I. Buvat, "Quantification in simultaneous $99 \mathrm{mTc} / 123 \mathrm{I}$ brain SPECT using generalized spectral factor analysis: a Monte Carlo study," Physics in Medicine and Biology, vol. 51, no. 23, pp. 6157-6171, 2006.

[25] A. M. Blamire, "The technology of MRI: the next 10 years?" British Journal of Radiology, vol. 81, no. 968, pp. 601-617, 2008.

[26] J. C. Gore, H. C. Manning, C. C. Quarles, K. W. Waddell, and T. E. Yankeelov, "Magnetic resonance in the era of molecular imaging of cancer," Magnetic Resonance Imaging, vol. 29, no. 5, pp. 587-600, 2011.

[27] P. L. Rapley, C. Witiw, K. Rich et al., "In vitro molecular magnetic resonance imaging detection and measurement of apoptosis using superparamagnetic iron oxide + antibody as ligands for nucleosomes," Physics in Medicine and Biology, vol. 57, no. 21, pp. 7015-7028, 2012.

[28] I. Debergh, N. Van Damme, D. De Naeyer et al., "Molecular imaging of tumor-associated angiogenesis using a novel magnetic resonance imaging contrast agent targeting $\alpha \mathrm{v} \beta 3$ integrin," Annals of Surgical Oncology, 2013.

[29] S. Sawall, J. Kuntz, M. Socher et al., "Imaging of cardiac perfusion of free-breathing small animals using dynamic phasecorrelated micro-CT," Medical Physics, vol. 39, no. 12, pp. 74997506, 2012.

[30] S. M. Johnston, G. A. Johnson, and C. T. Badea, “Temporal and spectral imaging with micro-CT," Medical Physics, vol. 39, no. 8, pp. 4943-4958, 2012.

[31] J. Schaap, J. A. de Groot, K. Nieman et al., "Hybrid myocardial perfusion SPECT/CT coronary angiography and invasive coronary angiography in patients with stable angina pectoris lead to similar treatment decisions," Heart, vol. 99, no. 3, pp. 188-194, 2013.

[32] R. Popovtzer, A. Agrawal, N. A. Kotov et al., "Targeted gold nanoparticles enable molecular CT imaging of cancer," Nano Letters, vol. 8, no. 12, pp. 4593-4596, 2008.

[33] F. Hyafil, J.-C. Cornily, J. E. Feig et al., "Noninvasive detection of macrophages using a nanoparticulate contrast agent for computed tomography," Nature Medicine, vol. 13, no. 5, pp. 636-641, 2007.

[34] D. Pan, T. A. Williams, A. Senpan et al., "Detecting vascular biosignatures with a colloidal, radio-opaque polymeric nanoparticle," Journal of the American Chemical Society, vol. 131, no. 42, pp. 15522-15527, 2009.

[35] J. Li, A. Chaudhary, S. J. Chmura et al., "A novel functional CT contrast agent for molecular imaging of cancer," Physics in Medicine and Biology, vol. 55, no. 15, pp. 4389-4397, 2010.

[36] M. Rotman, T. J. A. Snoeks, and L. van der Weerd, "Pre-clinical optical imaging and MRI for drug development in Alzheimer's disease," Drug Discovery Today, vol. 8, no. 2-4, pp. el17-e125, 2011.

[37] L. E. Edgington, A. B. Berger, G. Blum et al., "Noninvasive optical imaging of apoptosis by caspase-targeted activity-based probes," Nature Medicine, vol. 15, no. 8, pp. 967-973, 2009. 
[38] G. Niu, L. Zhu, D. N. Ho et al., "Longitudinal bioluminescence imaging of the dynamics of Doxorubicin induced apoptosis," Theranostics, vol. 3, no. 3, pp. 190-200, 2013.

[39] R. Atreya, M. J. Waldner, and M. F. Neurath, "Molecular imaging: interaction between basic and clinical science," Gastroenterology Clinics of North America, vol. 39, no. 4, pp. 911-922, 2010.

[40] J. Bzyl, W. Lederle, M. Palmowski, and F. Kiessling, "Molecular and functional ultrasound imaging of breast tumors," European Journal of Radiology, vol. 81, supplement 1, pp. S11-S12, 2012.

[41] N. Deshpande, A. Needles, and J. K. Willmann, "Molecular ultrasound imaging: current status and future directions," Clinical Radiology, vol. 65, no. 7, pp. 567-581, 2010.

[42] P. Li, Y. Zheng, H. Ran et al., "Ultrasound triggered drug release from 10-hydroxycamptothecin-loaded phospholipid microbubbles for targeted tumor therapy in mice," Journal of Controlled Release, vol. 162, no. 2, pp. 349-354, 2012.

[43] J. T. Sutton, K. J. Haworth, G. Pyne-Geithman, and C. K. Holland, "Ultrasound-mediated drug delivery for cardiovascular disease," Expert Opinion on Drug Delivery, vol. 10, no. 5, pp. 573592, 2013.

[44] M. Mancini, A. Greco, G. Salvatore et al., "Imaging of thyroid tumor angiogenesis with microbubbles targeted to vascular endothelial growth factor receptor type 2 in mice," BMC Medical Imaging, vol. 13, article 31, 2013.

[45] P. Li, Y. Zheng, H. Ran et al., "Ultrasound triggered drug release from 10-hydroxycamptothecin-loaded phospholipid microbubbles for targeted tumor therapy in mice," Journal of Controlled Release, vol. 162, no. 2, pp. 349-354, 2012.

[46] Z.-Y. Chen, K. Liang, and R. X. Qiu, “Targeted gene delivery in tumor xenografts by the combination of ultrasound-targeted microbubble destruction and polyethylenimine to inhibit survivin gene expression and induce apoptosis," Journal of Experimental and Clinical Cancer Research, vol. 29, no. 1, pp. 1-9, 2010.

[47] W. Cai and X. Chen, "Nanoplatforms for targeted molecular imaging in living subjects," Small, vol. 3, no. 11, pp. 1840-1854, 2007.

[48] Y. Lin, Z. Y. Chen, and F. Yang, "Ultrasound-based multimodal molecular imaging and functional ultrasound contrast agents," Current Pharmaceutical Design, vol. 19, no. 18, pp. 3342-3351, 2013.

[49] T. D. Poeppel, B. J. Krause, T. A. Heusner, C. Boy, A. Bockisch, and G. Antoch, "PET/CT for the staging and follow-up of patients with malignancies," European Journal of Radiology, vol. 70, no. 3, pp. 382-392, 2009.

[50] W. Cai, G. Niu, and X. Chen, "Multimodality imaging of the HER-kinase axis in cancer," European Journal of Nuclear Medicine and Molecular Imaging, vol. 35, no. 1, pp. 186-208, 2008.

[51] W. Cai and X. Chen, "Multimodality molecular imaging of tumor angiogenesis," The Journal of Nuclear Medicine, vol. 49, supplement 2, pp. 113S-128S, 2008.

[52] Z. S. Browning, A. A. Wilkes, D. S. Mackenzie et al., "Using PET/ CT imaging to characterize 18 F-fluorodeoxyglucose utilization in fish," Journal of Fish Diseases, vol. 36, no. 11, pp. 911-919, 2013.

[53] R. Abgral, S. Querellou, G. Potard et al., "Does 18F-FDG PET/ CT improve the detection of posttreatment recurrence of head and neck squamous cell carcinoma in patients negative for disease on clinical follow-up?" Journal of Nuclear Medicine, vol. 50, no. 1, pp. 24-29, 2009.

[54] W. W. Lee, B. Marinelli, A. M. Van Der Laan et al. et al., "PET/ MRI of inflammation in myocardial infarction," Journal of the American College of Cardiology, vol. 59, no. 2, pp. 153-163, 2012.
[55] A. Kjær, A. Loft, I. Law et al., "PET/MRI in cancer patients: first experiences and vision from Copenhagen," Magnetic Resonance Materials in Physics, Biology and Medicine, vol. 26, no. 1, pp. 3747, 2012.

[56] B. Li, M. Abran, C. Matteau-Pelletier et al., "Low-cost threedimensional imaging system combining fluorescence and ultrasound," Journal of Biomedical Optics, vol. 16, no. 12, Article ID 126010, 2011.

[57] P. Agrawal, G. J. Strijkers, and K. Nicolay, "Chitosan-based systems for molecular imaging," Advanced Drug Delivery Reviews, vol. 62, no. 1, pp. 42-58, 2010.

[58] M. A. McDonald, P. C. Wang, and E. L. Siegel, "Protein nanospheres: synergistic nanoplatform-based probes for multimodality imaging," in Reporters, Markers, Dyes, Nanoparticles, and Molecular Probes for Biomedical Applications III, Proceedings of SPIE, San Francisco, Calif, USA, January 2011.

[59] G. D. Kenny, N. Kamaly, T. L. Kalber et al., "Novel multifunctional nanoparticle mediates siRNA tumour delivery, visualisation and therapeutic tumour reduction in vivo," Journal of Controlled Release, vol. 149, no. 2, pp. 111-116, 2011.

[60] L. Evangelista, A. R. Cervino, S. Chondrogiannis et al., "Comparison between anatomical cross-sectional imaging and 18FFDG PET/CT in the staging, restaging, treatment response, and long-term surveillance of squamous cell head and neck cancer: a systematic literature overview," Nuclear Medicine Communications, vol. 35, no. 2, pp. 123-134, 2014.

[61] B. X. Ren, F. Yang, G. H. Zhu et al., "Magnetic resonance tumor targeting imaging using gadolinium labeled human telomerase reverse transcriptase antisense probes," Cancer Science, vol. 103, no. 8, pp. 1434-1439, 2012.

[62] J. Gao and H. Zheng, "Illuminating the lipidome to advance biomedical research: peptide-based probes of membrane lipids," Future Medicinal Chemistry, vol. 5, no. 8, pp. 947-959, 2013.

[63] L. Hansen, E. K. Unmack Larsen, E. H. Nielsen et al., “Targeting of peptide conjugated magnetic nanoparticles to urokinase plasminogen activator receptor (uPAR) expressing cells," Nanoscale, vol. 5, no. 17, pp. 8192-8201, 2013.

[64] B. J. Hackel, R. H. Kimura, and Z. Miao, "18F-Fluorobenzoatelabeled cystine knot peptides for PET imaging of integrin $\alpha \mathrm{v} \beta 6$," Nanoscale, vol. 55, no. 7, pp. 1101-1105, 2013.

[65] S. Oliveira, R. Heukers, J. Sornkom, R. J. Kok, and P. M. van Bergen en Henegouwen, "Targeting tumors with nanobodies for cancer imaging and therapy," Journal of Controlled Release, vol. 172, no. 3, pp. 607-617, 2013.

[66] Y. Zhang, H. Hong, and H. Orbay, "PET imaging of CD105/ endoglin expression with a ${ }^{61 / 64} \mathrm{Cu}$-labeled $\mathrm{Fab}$ antibody fragment," European Journal of Nuclear Medicine and Molecular Imaging, vol. 40, no. 5, pp. 759-767, 2013.

[67] M. Abdolahi, D. Shahbazi-Gahrouei, S. Laurent et al., "Synthesis and in vitro evaluation of MR molecular imaging probes using J591 mAb-conjugated SPIONs for specific detection of prostate cancer," Contrast Media \& Molecular Imaging, vol. 8, no. 2, pp. 175-184, 2013.

[68] P. R. Bouchard, R. M. Hutabarat, and K. M. Thompson, "Discovery and development of therapeutic aptamers," Annual Review of Pharmacology and Toxicology, vol. 50, pp. 237-257, 2010.

[69] A. D. Keefe, S. Pai, and A. Ellington, "Aptamers as therapeutics," Nature Reviews Drug Discovery, vol. 9, no. 7, pp. 537-550, 2010.

[70] H. Hong, S. Goel, Y. Zhang, and W. Cai, "Molecular imaging with nucleic acid aptamers," Current Medicinal Chemistry, vol. 18, no. 27, pp. 4195-4205, 2011. 
[71] H. Shi, X. He, K. Wang et al., "Activatable aptamer probe for contrast-enhanced in vivo cancer imaging based on cell membrane protein-triggered conformation alteration," Proceedings of the National Academy of Sciences of the United States of America, vol. 108, no. 10, pp. 3900-3905, 2011.

[72] V. Bagalkot, L. Zhang, E. Levy-Nissenbaum et al., "Quantum dot-aptamer conjugates for synchronous cancer imaging, therapy, and sensing of drug delivery based on Bi-fluorescence resonance energy transfer," Nano Letters, vol. 7, no. 10, pp. 30653070, 2007.

[73] D. W. Hwang, H. Y. Ko, J. H. Lee et al., "A nucleolin-targeted multimodal nanoparticle imaging probe for tracking cancer cells using an aptamer," Journal of Nuclear Medicine, vol. 51, no. 1, pp. 98-105, 2010.

[74] A. Louie, "Multimodality imaging probes: design and challenges," Chemical Reviews, vol. 110, no. 5, pp. 3146-3195, 2010.

[75] M. A. Hall, S. Kwon, H. Robinson et al., "Imaging prostate cancer lymph node metastases with a multimodality contrast agent," Prostate, vol. 72, no. 2, pp. 129-146, 2012.

[76] S. Y. Madani, F. Shabani, M. V. Dwek et al., "Conjugation of quantum dots on carbon nanotubes for medical diagnosis and treatment," International Journal of Nanomedicine, vol. 8, pp. 941-950, 2013.

[77] R. John, F. T. Nguyen, K. J. Kolbeck et al., “Targeted multifunctional multimodal protein-shell microspheres as cancer imaging contrast agents," Molecular Imaging and Biology, vol. 14, pp. 17-24, 2012.

[78] J. Ma, P. Huang, M. He et al., "Folic acid-conjugated LaF3:Yb,Tm@SiO2 nanoprobes for targeting dual-modality imaging of upconversion luminescence and X-ray computed tomography," The Journal of Physical Chemistry B, vol. 116, no. 48, pp. 14062-14070, 2012.

[79] Z. Liu, T. Lammers, J. Ehling et al., "Iron oxide nanoparticlecontaining microbubble composites as contrast agents for MR and ultrasound dual-modality imaging," Biomaterials, vol. 32, no. 26, pp. 6155-6163, 2011.

[80] M. Liang, X. Liu, D. Cheng et al., "Multimodality nuclear and fluorescence tumor imaging in mice using a streptavidin nanoparticle," Bioconjugate Chemistry, vol. 21, no. 7, pp. 1385-1388, 2010.

[81] J. K. Willmann, Z. Cheng, C. Davis et al., “Targeted microbubbles for imaging tumor angiogenesis: assessment of whole-body biodistribution with dynamic micro-pet in mice," Radiology, vol. 249, no. 1, pp. 212-219, 2008.

[82] N. Deshpande, A. Needles, and J. K. Willmann, "Molecular ultrasound imaging: current status and future directions," Clinical Radiology, vol. 65, no. 7, pp. 567-581, 2010.

[83] F. Yan, X. Li, Q. Jin et al., “Therapeutic ultrasonic microbubbles carrying paclitaxel and LyP-1 peptide: preparation, characterization and application to ultrasound-assisted chemotherapy in breast cancer cells," Ultrasound in Medicine and Biology, vol. 37, no. 5, pp. 768-779, 2011. 


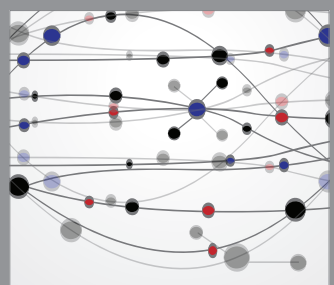

The Scientific World Journal
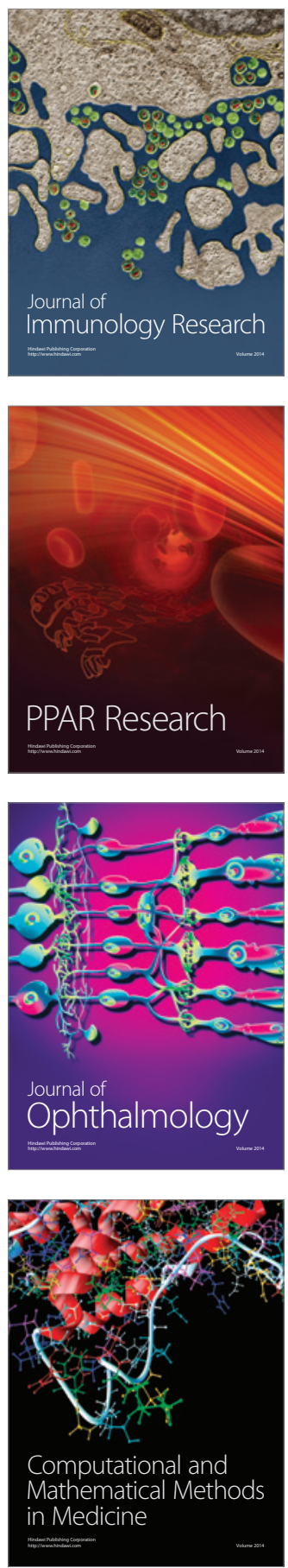

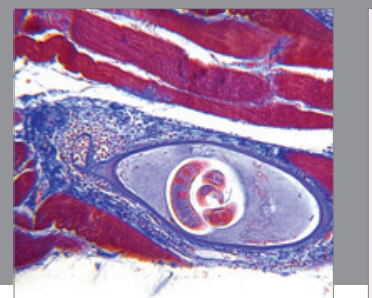

Gastroenterology

Research and Practice
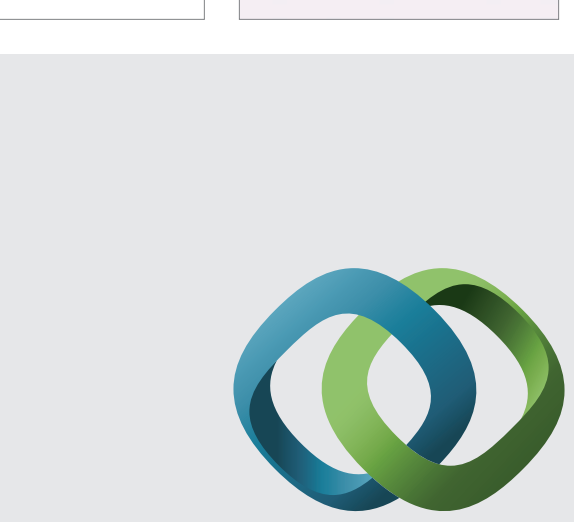

\section{Hindawi}

Submit your manuscripts at

http://www.hindawi.com
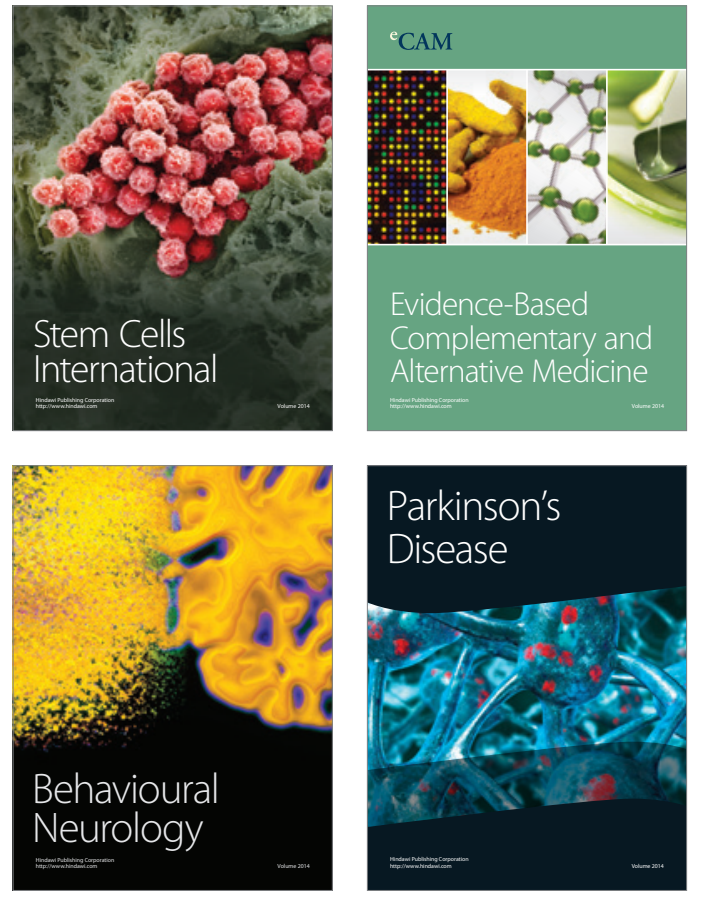
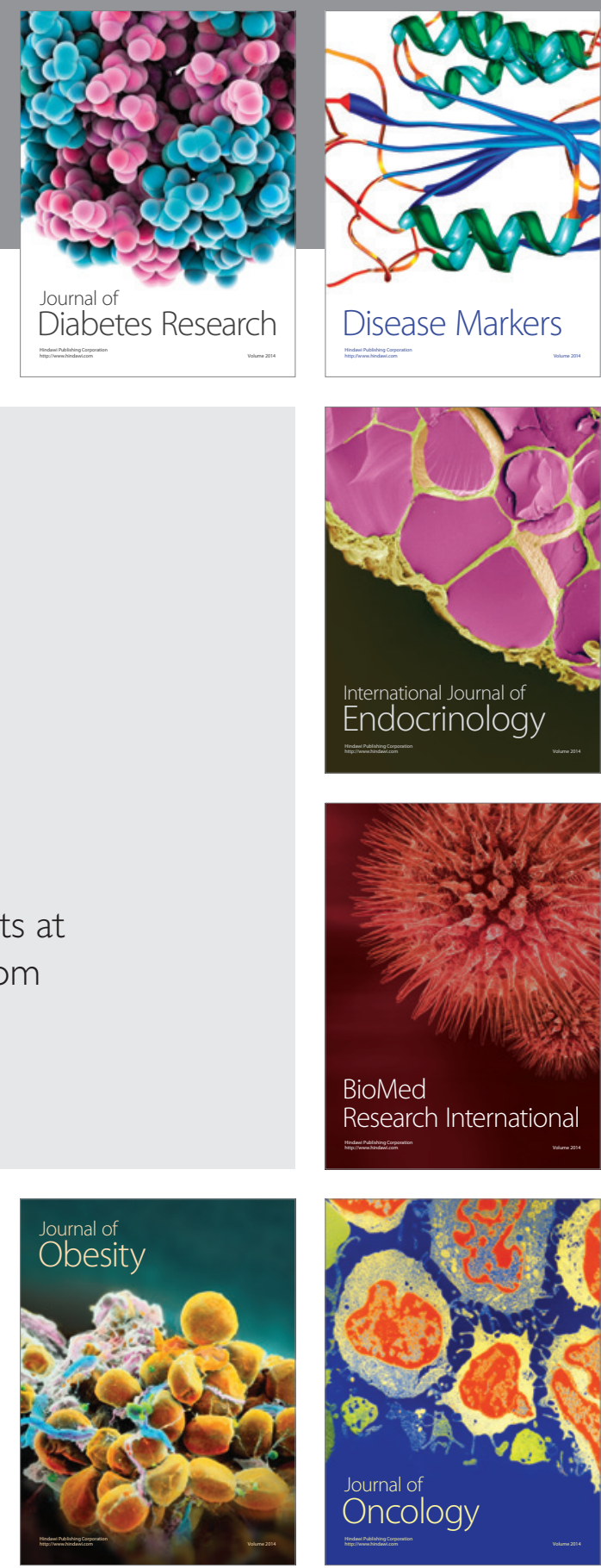

Disease Markers
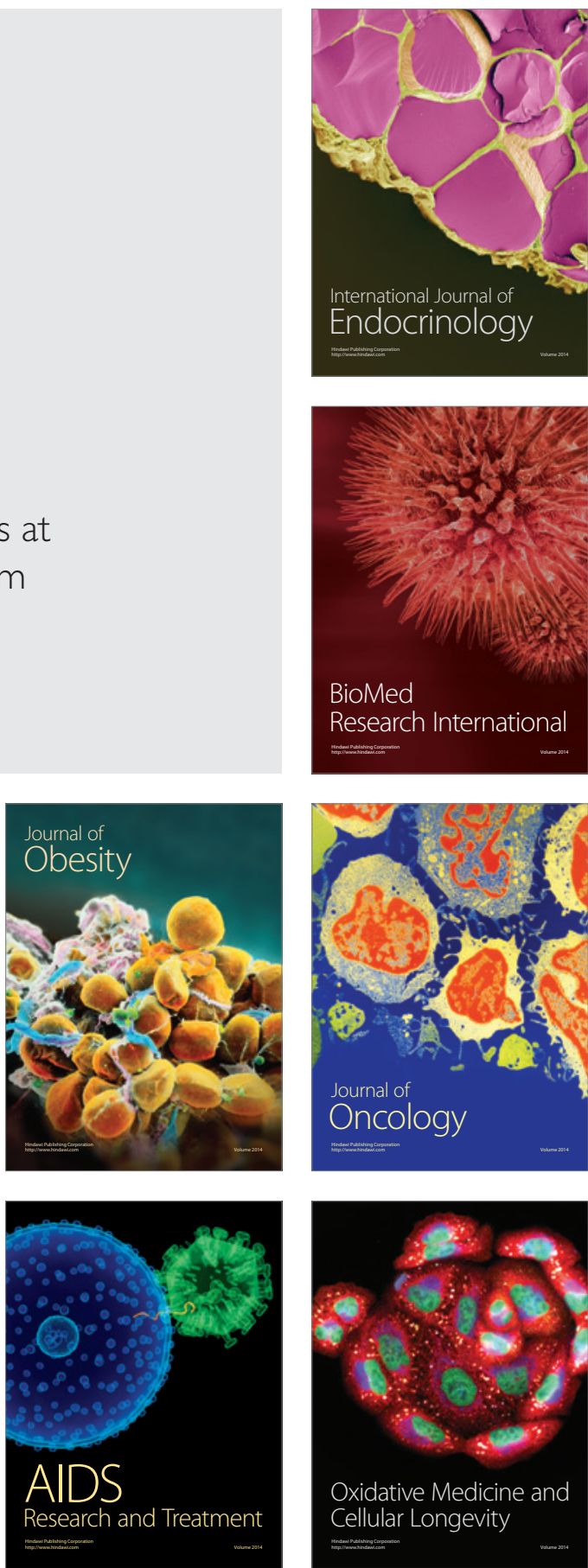